\title{
Predicting Cardiovascular Risk Using Social Media Data: Performance Evaluation of Machine-Learning Models
}

Anietie U Andy ${ }^{1}$, PhD; Sharath C Guntuku ${ }^{1,2}$, PhD; Srinath Adusumalli3 ${ }^{3,4}$, MD, MSc; David A Asch ${ }^{3,5,6}$, MD, MBA; Peter W Groeneveld ${ }^{5,6}$, MD, MS; Lyle H Ungar ${ }^{1}$, PhD; Raina M Merchant ${ }^{1,3,7}$, MD, MSHP

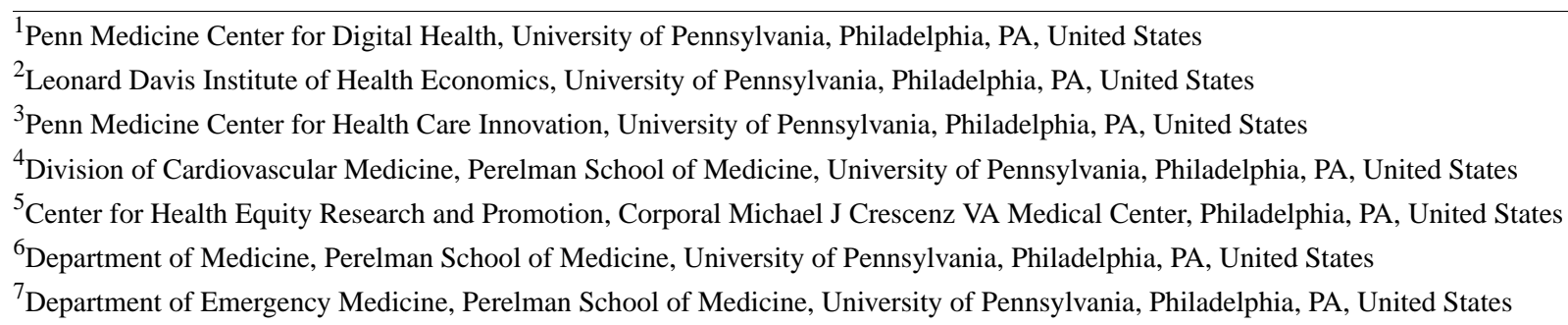

Corresponding Author:

Anietie U Andy, $\mathrm{PhD}$

Penn Medicine Center for Digital Health

University of Pennsylvania

Philadelphia, PA

United States

Phone: 12024864095

Email: Anietie.Andy@pennmedicine.upenn.edu

\section{Abstract}

Background: Current atherosclerotic cardiovascular disease (ASCVD) predictive models have limitations; thus, efforts are underway to improve the discriminatory power of ASCVD models.

Objective: We sought to evaluate the discriminatory power of social media posts to predict the 10-year risk for ASCVD as compared to that of pooled cohort risk equations (PCEs).

Methods: We consented patients receiving care in an urban academic emergency department to share access to their Facebook posts and electronic medical records (EMRs). We retrieved Facebook status updates up to 5 years prior to study enrollment for all consenting patients. We identified patients $(\mathrm{N}=181)$ without a prior history of coronary heart disease, an ASCVD score in their EMR, and more than 200 words in their Facebook posts. Using Facebook posts from these patients, we applied a machine-learning model to predict 10-year ASCVD risk scores. Using a machine-learning model and a psycholinguistic dictionary, Linguistic Inquiry and Word Count, we evaluated if language from posts alone could predict differences in risk scores and the association of certain words with risk categories, respectively.

Results: The machine-learning model predicted the 10-year ASCVD risk scores for the categories $<5 \%, 5 \%-7.4 \%, 7.5 \%-9.9 \%$, and $\geq 10 \%$ with area under the curve (AUC) values of $0.78,0.57,0.72$, and 0.61 , respectively. The machine-learning model distinguished between low risk $(<10 \%)$ and high risk $(>10 \%)$ with an AUC of 0.69. Additionally, the machine-learning model predicted the ASCVD risk score with Pearson $r=0.26$. Using Linguistic Inquiry and Word Count, patients with higher ASCVD scores were more likely to use words associated with sadness $(r=0.32)$.

Conclusions: Language used on social media can provide insights about an individual's ASCVD risk and inform approaches to risk modification.

(JMIR Cardio 2021;5(1):e24473) doi: 10.2196/24473

\section{KEYWORDS}

ASCVD; machine learning; natural language processing; atherosclerotic; cardiovascular disease; social media language; social media 


\section{Introduction}

Secondary prevention approaches have improved the longevity of patients with cardiovascular (CV) disease; however, risk factors and adverse health behaviors (eg, physical inactivity, smoking) are highly prevalent, and $<1 \%$ of adults in most contemporary series meet all factors of ideal CV health [1]. The logistics and practicalities of meeting the goal of ideal CV health have not been clearly elucidated. Practice guidelines recommend using atherosclerotic cardiovascular disease (ASCVD) pooled cohort equations (PCEs) [2] or other prediction tools to classify patients' risk of $\mathrm{CV}$ disease and the need for risk-reducing therapies such as statin medications [3]. There is also an increasing focus on identifying markers that provide better measures of risk. To best prevent ASCVD, it is important to precisely determine an individual's 10-year risk for ASCVD. As digital platforms are increasingly used to document health behaviors, data from digital sources may provide a window into manifestations of novel risk factors and can provide complementary data to characterize existing risk factors.

Social media data in the form of posts, photos, and "likes" can provide information about individuals' daily activities and behaviors. Social media has been used to track heart disease mortality rates [4] and depression [5]. Data on social media platforms are generated at a fast rate. Accessing these data from consenting individuals offers an opportunity to collect and analyze these data in real time. This information could facilitate identification of earlier signals of disease development or exacerbation, and timely tracking of the health of individuals and the collective health of a community [4-9]. The data are generally unscripted and spontaneous, and can therefore provide information that is different from standard survey assessments. Another potential use of data from digital platforms is that they can be used for direct intervention, so that the same platforms that are being used to assess insights can also be used to deliver targeted health information or evaluate information delivery.

The potential of social media data for $\mathrm{CV}$ health lies in tracking, codifying, and better understanding the hard-to-measure lifestyle choices, along with exposures related to diet, exercise, smoking, and other factors that can significantly contribute to the development and progression of heart disease. At present, measuring many of these behaviors is dependent on self-report and recall [4]. Yet, posts or images from digital media could better inform a patient-provider discussion about how to change actual dietary choices and consumption. Incorporating data from digital sources has the potential to enhance our approach for characterizing individuals' risk and tailoring management, as a new type of precision medicine.

We sought to use social media data from consenting individuals to predict ASCVD risk reported in an electronic medical record (EMR), and to characterize differences in posts relative to four categories based on the 10-year primary risk from ASCVD risk scores.

\section{Methods}

\section{Data and Design}

This was a retrospective analysis of social media and EMR data of consenting patients. This study was approved by the University of Pennsylvania Institutional Review Board.

Recruitment for compiling the social mediome dataset began in March 2014 and included patients from inpatient and outpatient settings across two urban academic medical centers. Participants in this dataset consented to sharing access for selecting historical data from their social media accounts (eg, Facebook, Twitter, Instagram) and access to their medical record data. Data are stored in a Health Insurance Portability and Accountability Act (HIPAA)-compliant secure server and participants can elect to discontinue sharing data at any time point. Additional information about this dataset is published elsewhere [10,11].

Following recommendations of Goff et al [12], which outlines the process of developing a risk equation for predicting the 10-year ASCVD risk of individuals between the ages of 40 and 79 years, from our dataset, we identified patients aged 40 to 79 years and without a prior history of ASCVD documented in their EMR. Of these patients, we identified 181 with a calculated 10 -year primary risk of ASCVD score in their EMR. For all these patients, demographics (age, race, gender) were also extracted from the EMR along with ASCVD scores. We retrieved Facebook status updates up to 5 years prior to enrollment for all users.

Table 1 shows the demographic information of patients included in our analysis. Of the 181 participants meeting the criteria for this study, the majority were women and Black, and the average age was 50 years. The participants had 159,958 Facebook posts overall (mean 884, SD 3227).

Table 1. Demographic information of patients included in our analysis $(\mathrm{N}=181)$.

\begin{tabular}{ll}
\hline Characteristic & Value \\
\hline Race, $\mathbf{n}(\%)$ & \\
$\quad$ African American & $104(57.5)$ \\
White & $64(35.4)$ \\
Other & $13(7.2)$ \\
Men, $\mathrm{n}(\%)$ & $48(26.5)$ \\
\hline
\end{tabular}

We used two approaches to process language from social media posts for inclusion in a regression model. Specifically, language features from posts were derived using (a) open vocabulary topics and (b) dictionary-based psycholinguistic features. These derived language features were then used to predict the patients' 
10-year ASCVD risk scores and to distinguish patients with different ASCVD risk scores.

\section{Open Vocabulary Approach}

The open vocabulary approach uses latent Dirichlet allocation (LDA) [13], which is a natural language-processing method that is used to analyze the co-occurrences of words in text (in this case Facebook posts). Distinct groupings of these words represent topics (eg, groups of co-occurring words) and these topics can be labeled based on their content. For example, the model could cluster the words "dinner," "cheese," "eat," "made," and "food" as a reference to food by utilizing the similarities in the distributional properties in the Facebook posts. We generated 20 topics using Facebook posts from all of the users in our dataset. Each user was represented as a 20-dimensional vector based on the probability of each topic in all users' posts. Multimedia Appendix 1 shows the LDA topics and 10 words associated with each topic. To determine the number of topics, consistent with prior work [14,15], we varied the number of topics using 10, 20, 50, 75, and 100 topics, respectively; 20 topics had the most coherent topic themes when reviewed by one of the coauthors.

\section{Dictionary-Based Approach}

The dictionary-based psycholinguistic approach uses language from Facebook posts to identify the prevalence of predefined word categories represented in the Linguistic Inquiry and Word Count (LIWC) dictionary [16]. LIWC represents a dictionary of 73 different psycholinguistic word categories such as topical categories and emotions. For each user, the rate of words that occurred in a given LIWC category was measured and included as input in a model to predict ASCVD risk as described below.

\section{Predicting ASCVD Risk Scores Using Social Media Language}

We sought to investigate the discriminatory power of predicting a patient's 10-year ASCVD risk using language features derived from Facebook posts. We extracted the features described using an open-vocabulary approach and trained a logistic regression model, as implemented in Python 3.4 scikit-learn [17], to predict ASCVD risk scores using 5-fold cross-validation. We defined the outcome in three different ways.
In 2013, the American Heart Association and American College of Cardiology put forth the ASCVD PCEs [2], which can be used to predict an individual's 10-year risk of ASCVD. Therefore, in Model 1, ASCVD risk was set as a categorical variable. We categorized patients into the following different thresholds: $<5 \%, 5 \%-7.4 \%, 7.5 \%-9.9 \%$, and $\geq 10 \%$. We trained a multiclass logistic predictive model to predict these four categories of ASCVD risk scores. The prediction performance is reported as the area under the receiver operating characteristic curve (AUC).

For Model 2, the ASCVD risk score of patients was applied as a continuous variable rather than as ategorical variable that was used in Model 1. The performance of Model 2 was assessed using the Pearson correlation coefficient $(r)$.

Identifying patients with high risk $(\geq 10 \%)$ of ASCVD is of interest to clinicians. Therefore, in Model 3, we treated ASCVD risk as a dichotomous variable and built a logistic regression model to distinguish the high-risk category using language compared to low ASCVD scores (ie, <10\%). Additionally, we used LIWC to distinguish the different features associated with high-risk patients by correlating the LIWC category feature of patients from their social media posts and whether they are in the high-risk $(>10 \%)$ or low-risk $(<10 \%)$ categories; we measured the effect size using Cohen $d$. To indicate significant correlations, we used Benjamini-Hochberg $P$ value correction with a significance threshold of $P<.001$.

\section{Results}

\section{Predicting ASCVD Risk Score Using Social Media Language}

\section{Model 1}

The multiclass logistic regression model on Facebook posts was trained to classify patients in four different categories $(<5 \%$, $5 \%-7.4 \%, 7.5 \%-9.9 \%, \geq 10 \%)$ based on their ASCVD risk scores. The model was able to delineate patients in the lowest risk category $(<5 \%)$ from patients in other categories with an AUC of 0.78 . The model delineated patients in the categories $5 \%-7.4 \%, 7.5 \%-9.9 \%$, and $\geq 10 \%$ from those in other categories, as shown in Table 2.

Table 2. Area under the curve (AUC) scores for each category of atherosclerotic cardiovascular disease risk scores from Model 1.

\begin{tabular}{lll}
\hline Category & AUC (age only) & AUC (language only) \\
\hline$<5 \%$ & 0.52 & 0.78 \\
$5 \%-7.4 \%$ & 0.55 & 0.57 \\
$7.5 \%-9.9 \%$ & 0.45 & 0.72 \\
$\geq 10 \%$ & 0.59 & 0.61 \\
\hline
\end{tabular}

\section{Model 2}

Using the linear regression model on Facebook posts, we predicted the ASCVD risk score of patients with $r=0.26$ $(P<.001)$.

\section{Model 3}

The logistic regression model delineated patients with a high risk $(\geq 10 \%)$ of ASCVD from those with a low risk $(<10 \%)$ with an AUC of 0.69 . 


\section{Identifying Differentially Expressed Language Features According to High and Low ASCVD Scores}

The sadness LIWC category was most strongly associated with the high ASCVD risk category $(\geq 10 \%)$ at a Benjamini-Hochberg-corrected significance level of $P<.001$ and Pearson $r=0.32$. None of the other LDA topics or LIWC categories was significantly associated with high and low ASCVD risk.

\section{Discussion}

\section{Principal Findings}

Language from Facebook posts has the potential to distinguish patients based on their calculated 10-year ASCVD risk score categorization and actual risk score. Although social media data are unlikely to replace traditional approaches for predicting $\mathrm{CV}$ risk, these findings suggest that such data can potentially provide supplemental information about an individual's lifestyle and behavior, which can complement our understanding of contributors to long-term CV risk. More than 2 billion people share information about their daily lives on social media platforms, which can include information about what they eat and drink, if they smoke, when they exercise, what their lab results are, and other factors associated with Life's Simple 7 [18]. However, less is known about how much of this information is noise or if there is an actual relevant signal in the volumes of data in online chatter such as Facebook, where individuals often reveal information about themselves. Additionally, prior work has demonstrated that social media data can be used to predict several medical conditions such as diabetes and mental health conditions [4,5].

The potential opportunity in exploring social media data is that this emerging data source could include data about behavior and lifestyle that might not have been reported to clinicians. There is still a gap in how this would be implemented in clinical practice, and would require further evaluation of feasibility, acceptability, and interpretability. These data are unlikely to replace the existing risk score input but rather may provide complementary adjunct data. Prior work has explored the contribution of nonclinical factors (eg, patient interviews about socioeconomic status, health status, adherence, psychosocial characteristics) in predicting CV outcomes (eg, congestive heart failure readmissions). The model performance overall was poor, although patient-reported information extended the predicted ranges of rates of readmission and slightly improved model discrimination [19]. Social media data in the form of photos, videos, and likes [20,21] have been used to predict users' personality [22], mental health, and other behaviors. Consequently, future work could use multiple modalities of user-generated content to model the ASCVD risk score.
In our patient cohort, a high ASCVD risk score was associated with increased use of "sad" language on Facebook. This is consistent with research demonstrating that depression is more prevalent in populations with $\mathrm{CV}$ disease, and is predictive of adverse outcomes (such as myocardial infarction and death) among populations with preexisting CV disease [23].

In our analysis, the AUC for Model 1 indicated low accuracy. A potential reason for this is that we used data from individuals between the ages of 40 and 79 years, and individuals in this age group do not post as much on social media compared to younger individuals. Accordingly, in our dataset, some users had fewer posts, leading to low accuracy from the AUC. We hypothesize that with more posts (ie, more words), our models will perform better.

We compared Models 1 and 3 together to determine which performed better at predicting the ASCVD risk score of individuals. Toward this end, we computed the micro AUC of Model 1 and compared it to that of Model 3, which was 0.66 and 0.69 , respectively. This suggests that Model 3 is more reliable at predicting ASCVD risk compared to Model 1.

The findings of this study offer promise for using emerging digital data sources for identifying risk factors. This moves beyond what is simply reported by patients to what may be revealed when looking at a diary of information over multiple time points. This could aid clinicians in providing individualized recommendations for managing risk factors that contribute to heart disease.

\section{Limitations}

This study has several limitations. The study cohort was primarily female and African American. Our analysis used posts from patients with at least 200 words in their Facebook posts, and therefore we cannot extrapolate about those who used social media less or did not consent to share; we used 200 words because prior work on using social media for predicting individuals' traits determined that for good and stable predictive performance when working with social media data, data from users with 200 words or more on Facebook should be used [24,25]. Our sample was also limited to those with an ASCVD risk score in a single health system EMR, and therefore we may have missed individuals with a risk score in another EMR or that may not have had a risk score calculated in our EMR.

\section{Conclusion}

We show that language from Facebook posts can be used to predict an individual's 10-year risk for ASCVD. Specific information in posts could help to guide clinicians in better understanding lifestyles and behaviors, and in counseling patients about heart disease risk.

\section{Acknowledgments}

This study is funded by the National Heart Lung and Blood Institute (NHLBI) of the National Institutes of Health (NIH) (1R01HL141844-01A1) to RM (principal investigator), DA, LA, and PG (coinvestigators). 


\section{Conflicts of Interest}

DA is a partner and part owner of VAL Health, and is a US government employee. The other authors have no conflicts of interest to declare.

\section{Multimedia Appendix 1}

Twenty topics generated from our dataset.

[DOCX File, 14 KB-Multimedia Appendix 1]

\section{References}

1. Ren J, Guo XL, Lu ZL, Zhang JY, Tang JL, Chen X, et al. Ideal cardiovascular health status and its association with socioeconomic factors in Chinese adults in Shandong, China. BMC Public Health 2016 Sep 07;16(1):942 [FREE Full text] [doi: 10.1186/s12889-016-3632-6] [Medline: 27605115]

2. Lloyd-Jones DM, Braun LT, Ndumele CE, Smith SC, Sperling LS, Virani SS, et al. Use of risk assessment tools to guide decision-making in the primary prevention of atherosclerotic cardiovascular disease: a special report from the American Heart Association and American College of Cardiology. J Am Coll Cardiol 2019 Jun 25;73(24):3153-3167. [doi: 10.1016/j.jacc.2018.11.005] [Medline: 30423392]

3. Yang X, Li J, Hu D, Chen J, Li Y, Huang J, et al. Predicting the 10-year risks of atherosclerotic cardiovascular disease in Chinese population: The China-PAR Project (Prediction for ASCVD Risk in China). Circulation 2016 Nov 08;134(19):1430-1440. [doi: 10.1161/CIRCULATIONAHA.116.022367] [Medline: 27682885]

4. Eichstaedt JC, Schwartz HA, Kern ML, Park G, Labarthe DR, Merchant RM, et al. Psychological language on Twitter predicts county-level heart disease mortality. Psychol Sci 2015 Feb 20;26(2):159-169 [FREE Full text] [doi: 10.1177/0956797614557867] [Medline: 25605707]

5. Merchant RM, Asch DA, Crutchley P, Ungar LH, Guntuku SC, Eichstaedt JC, et al. Evaluating the predictability of medical conditions from social media posts. PLoS One 2019 Jun 17;14(6):e0215476 [FREE Full text] [doi:

10.1371/journal.pone.0215476] [Medline: 31206534]

6. Young SD. Behavioral insights on big data: using social media for predicting biomedical outcomes. Trends Microbiol 2014 Nov;22(11):601-602 [FREE Full text] [doi: 10.1016/j.tim.2014.08.004] [Medline: 25438614]

7. Ahmed H, Younis EM, Hendawi A, Ali AA. Heart disease identification from patients' social posts, machine learning solution on Spark. Future Gener Comput Syst 2020 Oct;111:714-722. [doi: 10.1016/j.future.2019.09.056]

8. Young SD, Mercer N, Weiss RE, Torrone EA, Aral SO. Using social media as a tool to predict syphilis. Prev Med 2018 Apr;109:58-61 [FREE Full text] [doi: 10.1016/j.ypmed.2017.12.016] [Medline: 29278678]

9. Nguyen T, Larsen ME, O’Dea B, Nguyen DT, Yearwood J, Phung D, et al. Kernel-based features for predicting population health indices from geocoded social media data. Decis Support Syst 2017 Oct;102:22-31. [doi: 10.1016/j.dss.2017.06.010]

10. Asch DA, Rader DJ, Merchant RM. Mining the social mediome. Trends Mol Med 2015 Sep;21(9):528-529 [FREE Full text] [doi: 10.1016/j.molmed.2015.06.004] [Medline: 26341614]

11. Guntuku SC, Schwartz HA, Kashyap A, Gaulton JS, Stokes DC, Asch DA, et al. Author Correction: Variability in Language used on Social Media Prior to Hospital Visits. Sci Rep 2020 Jul 07;10(1):11456. [doi: 10.1038/s41598-020-68555-5] [Medline: 32632209]

12. Goff DC, Lloyd-Jones DM, Bennett G, Coady S, D'Agostino RB, Gibbons R, et al. 2013 ACC/AHA guideline on the assessment of cardiovascular risk: a report of the American College of Cardiology/American Heart Association Task Force on Practice Guidelines. J Am Coll Cardiol 2014 Jul 01;63(25 Pt B):2935-2959 [FREE Full text] [doi: 10.1016/j.jacc.2013.11.005] [Medline: 24239921]

13. Blei D, Ng A, Jordan M. Latent dirichllocation. J Machine Learn Res 2003;3:993-1022 [FREE Full text]

14. Ranard BL, Werner RM, Antanavicius T, Schwartz HA, Smith RJ, Meisel ZF, et al. Yelp reviews of hospital care can supplement and inform traditional surveys of the patient experience of care. Health Aff (Millwood) 2016 Apr;35(4):697-705 [FREE Full text] [doi: 10.1377/hlthaff.2015.1030] [Medline: 27044971]

15. Ryskina KL, Andy AU, Manges KA, Foley KA, Werner RM, Merchant RM. Association of Online Consumer Reviews of Skilled Nursing Facilities With Patient Rehospitalization Rates. JAMA Netw Open 2020 May 14;3(5):e204682. [doi: 10.1001/jamanetworkopen.2020.4682]

16. Pennebaker J, Boyd R, Jordan K, Blackburn K. The development and psychometric properties of LIWC2015. Austin, TX: University of Texas at Austin; 2015 Sep 01. URL: https://repositories.lib.utexas.edu/bitstream/handle/2152/31333/ LIWC2015 LanguageManual.pdf [accessed 2020-02-04]

17. Pedregosa F, Varoquaux G, Gramfort A, Michel V, Thirion B, Grisel O, et al. Scikit-learn: Machine learning in Python. J Machine Learn Res 2011 Oct 12;12:2825-2830 [FREE Full text]

18. Thacker EL, Gillett SR, Wadley VG, Unverzagt FW, Judd SE, McClure LA, et al. The American Heart Association Life's Simple 7 and incident cognitive impairment: The REasons for Geographic And Racial Differences in Stroke (REGARDS) study. J Am Heart Assoc 2014 Jun 11;3(3):e000635 [FREE Full text] [doi: 10.1161/JAHA.113.000635] [Medline: 24919926] 
19. Krumholz HM, Chaudhry SI, Spertus JA, Mattera JA, Hodshon B, Herrin J. Do non-clinical factors improve prediction of readmission risk?: Results from the Tele-HF Study. JACC Heart Fail 2016 Jan;4(1):12-20 [FREE Full text] [doi: 10.1016/j.jchf.2015.07.017] [Medline: 26656140]

20. Guntuku SC, Preotiuc-Pietro D, Eichstaedt JC, Ungar LH. What twitter profile and posted images reveal about depression and anxiety. 2019 Jun 11 Presented at: Proceedings of the Thirteenth International AAAI Conference on Web and Social Media (ICWSM 2019); 2019; Munich p. 236-246.

21. Abavisani M, Wu L, Hu S, Tetrault J, Jaimes A. Multimodal categorization of crisis events in social media. 2020 Presented at: Proceedings of the IEEE/CVF Conference on Computer Vision and Pattern Recognition; June 16, 2020; Virtual p. 14679-14689. [doi: 10.1109/cvpr42600.2020.01469]

22. Guntuku S, Lin W, Carpenter J, Ng WK, Ungar L. Studying personality through the content of posted and liked images on Twitter. 2017 Jun 01 Presented at: Proceedings of the 2017 ACM on web science conference; 2017; New York p. $223-227$. [doi: 10.1145/3091478.3091522]

23. Whooley MA, Wong JM. Depression and cardiovascular disorders. Annu Rev Clin Psychol 2013 Mar 28;9(1):327-354. [doi: 10.1146/annurev-clinpsy-050212-185526] [Medline: 23537487]

24. Jaidka K, Guntuku S, Ungar L. Facebook vs. twitter: Cross-platform differences in self-disclosure and trait prediction. 2018 Presented at: Proceedings of the Twelfth International AAAI Conference on Web and Social Media; 2018; California p. 141-150.

25. Eichstaedt JC, Smith RJ, Merchant RM, Ungar LH, Crutchley P, Preoţiuc-Pietro D, et al. Facebook language predicts depression in medical records. Proc Natl Acad Sci U S A 2018 Oct 30;115(44):11203-11208 [FREE Full text] [doi: 10.1073/pnas.1802331115] [Medline: $\underline{30322910]}$

\author{
Abbreviations \\ ASCVD: atherosclerotic cardiovascular disease \\ AUC: area under the receiver operating characteristic curve \\ CV: cardiovascular \\ EMR: electronic medical record \\ LDA: latent Dirichlet allocation \\ LIWC: Linguistic Inquiry and Word Count \\ PCE: pooled cohort equations
}

Edited by G Eysenbach; submitted 21.09.20; peer-reviewed by D Di Matteo, I Ahmed; comments to author 19.10.20; revised version
received 14.12.20; accepted 15.01.21; published 19.02.21
Please cite as:
Andy AU, Guntuku SC, Adusumalli S, Asch DA, Groeneveld PW, Ungar LH, Merchant RM
Predicting Cardiovascular Risk Using Social Media Data: Performance Evaluation of Machine-Learning Models
JMIR Cardio 2021;5(1):e24473
URL: $\underline{\text { http://cardio.jmir.org/2021/1/e24473/ }}$
doi: $\underline{10.2196 / 24473}$
PMID: $\underline{3605888}$

(C)Anietie U Andy, Sharath C Guntuku, Srinath Adusumalli, David A Asch, Peter W Groeneveld, Lyle H Ungar, Raina M Merchant. Originally published in JMIR Cardio (http://cardio.jmir.org), 19.02.2021. This is an open-access article distributed under the terms of the Creative Commons Attribution License (https://creativecommons.org/licenses/by/4.0/), which permits unrestricted use, distribution, and reproduction in any medium, provided the original work, first published in JMIR Cardio, is properly cited. The complete bibliographic information, a link to the original publication on http://cardio.jmir.org, as well as this copyright and license information must be included. 